\title{
Biocompatible and Light-Penetrating Hydrogels for Water Decontamination
}

\author{
Gloria Guidetti, Demetra Giuri, Nicola Zanna, Matteo Calvaresi, ${ }^{\circledR}$ Marco Montalti,* \\ and Claudia Tomasini**i(
}

Dipartimento di Chimica Giacomo Ciamician, Alma Mater Studiorum Università di Bologna, Via Selmi, 2, 40126 Bologna, Italy

\section{Supporting Information}

\begin{abstract}
Solar light-activated photocatalyst nanoparticles (NPs) are promising environment-friendly low cost tools for water decontamination, but their dispersion in the environment must be minimized. Here, we propose the incorporation of $\mathrm{TiO}_{2}$-NPs (also in combination with graphene platelets) into highly biocompatible hydrogels as a promising approach for the production of photoactive materials for water treatment. We also propose a convenient fluorescence-based method to investigate the hydrogel photocatalytic activity in real time with a conventional fluorimeter. Kinetics analysis of the degradation profile of a target fluorescent model pollutant demonstrates that fast

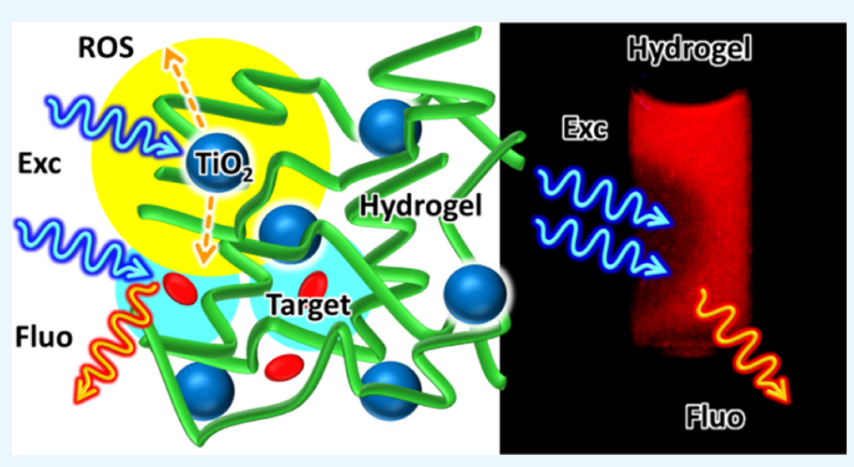
degradation occurs in the matrix bulk. Fluorescence anisotropy proved that small pollutant molecules diffuse freely in the hydrogel. Rheological and scanning electron microscopy characterization showed that the $\mathrm{TiO}_{2}-\mathrm{NP}$ incorporation does not significantly alter the hydrogel mechanical and morphological properties.
\end{abstract}

\section{INTRODUCTION}

Pollution is one of the most important unsolved problems that nowadays affects our society. Photocatalytic degradation of water pollutants is a very attractive strategy to convert contaminants into harmless compounds by using eco-friendly materials. ${ }^{1}$ This approach is typically based on nanostructured photocatalysts, which are activated by solar light and use the absorbed energy to produce reactive chemical species, starting from water and oxygen (as schematized in Figure 1), ${ }^{2}$ and are able to degrade organic pollutants. ${ }^{3,4}$ Most effective photocatalysts are metal-oxide semiconductors (such as $\mathrm{ZnO}, \mathrm{FeO}_{3}$, and $\mathrm{WO}_{3}{ }^{5}$ ) and, in particular, titanium dioxide nanoparticles $\left(\mathrm{TiO}_{2}-\mathrm{NPs}\right)$, in their anatase form, which have been widely used for a range of applications, including self-cleaning, ${ }^{5}$ surface sterilization, ${ }^{6}$ and air $^{7}$ and water ${ }^{8}$ purification. Advantageous features of $\mathrm{TiO}_{2}-\mathrm{NPs}$ are the excellent stability in water, large-scale availability, and low cost. $\mathrm{TiO}_{2}$-NPs also show low toxicity, ${ }^{9,10}$ nevertheless the recent concerns about the still partially unpredictable risks related to the impact of nanomaterials on the environment and human health, as a defensive approach to minimize their dispersion in the environment. ${ }^{11,12}$

Incorporation of $\mathrm{TiO}_{2}$-NPs in highly biocompatible, macroscopic matrixes that preserve the photocatalytic activity of the semiconductor NPs is hence a fundamental challenge in the design of new materials for photocatalysis. As a fundamental feature, these matrixes should present (i) a high water content because water plays an essential role in the photocatalytic process (see Figure 1), (ii) good permeability to small

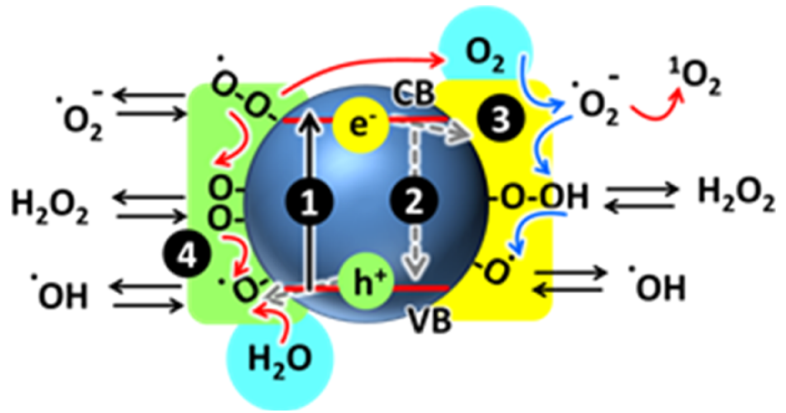

Figure 1. Reactive oxygen species generated in the photocatalytic reduction and oxidation steps of oxygen and water. Absorption of light (1) causes the transition of electrons to the conduction band and the formation of holes in the valence band. Part of the produced charges undergo recombination (2), whereas others migrate to the surface where trapped electrons (3) participate in the reductive steps (blue arrows) and trapped holes (4) in the oxidative processes.

molecules (pollutants), and (iii) good transparency to solar light.

In the last few years, we focused our research on the use of small pseudopeptides to form supramolecular hydrogels ${ }^{13-16}$ with specific properties, tailored to suit the applications of the

Received: May 17, 2018

Accepted: July 4, 2018

Published: July 19, 2018 
produced hydrogels. ${ }^{17-21}$ Hence, we could obtain hydrogels of different strengths, $\mathrm{pH}$ values, and transparencies. These properties are not all necessarily needed for a given application. In this paper, we want to describe the preparation of transparent hydrogels and their application for photodegradation. $^{22,23}$

We recently reported the preparation of hydrogels by slow $\mathrm{pH}$ variation of a water solution of Fmoc-L-Tyr-D-Oxd-OH $[$ Fmoc $=$ fluorenylmethyloxycarbonyl; $\mathrm{Tyr}=$ tyrosine; and $\mathrm{D}$ Oxd $=(4 R, 5 S)$-4-carboxy-5-methyl-oxazolidin-2-one $]$ ( $\mathbf{F}$ in Figure 2), ${ }^{24}$ and we compared their properties with the

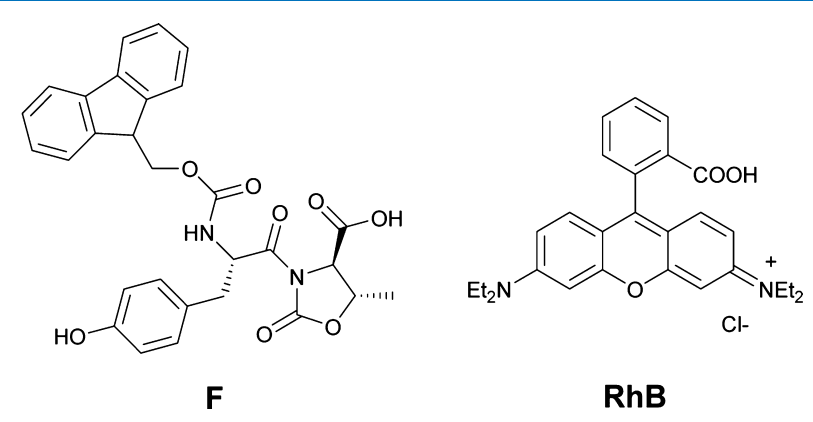

Figure 2. Chemical structure of gelator $\mathbf{F}$ and rhodamine RhB.

hydrogels formed with other gelators, among them Fmoc-PhePhe. ${ }^{25-28}$ We could demonstrate that the hydrogels prepared with gelator $\mathbf{F}$, with $\mathrm{pH}$ variation as a trigger, reach a high transparency. ${ }^{24}$ Here, we describe the preparation of hydrogels, based on $\mathbf{F}$, containing either $\mathrm{TiO}_{2}-\mathrm{NPs}$ or $\mathrm{TiO}_{2}-\mathrm{NPs}$ in combination with graphene platelets, and we demonstrate the ability to photodegrade a model pollutant compound (rhodamine B; RhB) upon ultraviolet (UV) irradiation. $\mathrm{TiO}_{2}-\mathrm{NPs}$, in fact, absorb the UV part of the solar spectrum $(\lambda<380$ $\mathrm{nm})^{29}$ being the band gap between the conduction band (CB) and the valence band (VB), ${ }^{10} \Delta E \approx 3.25 \mathrm{eV}$ (Figure 1). Integration of graphene in the photocatalytic $\mathrm{TiO}_{2}$ platform was also investigated to check the effect of graphene platelets on the hydrogel properties because carbon nanomaterials, ${ }^{30}$ such as nanotubes, ${ }^{31}$ carbon dots, ${ }^{32}$ graphene oxide, ${ }^{33}$ and reduced graphene oxide, ${ }^{34,35}$ have been reported to increase the photocatalytic performances of $\mathrm{TiO}_{2}-\mathrm{NPs}$.

We would like to stress that different from other systems where $\mathrm{TiO}_{2}-\mathrm{NPs}^{36,37}$ can be activated by sunlight only on the surface of the exposed material (e.g., $\mathrm{TiO}_{2}$ photocatalyst embedded in cementitious materials ${ }^{5,38-40}$ ), in our photocatalytic hydrogels, light penetrates inside the matrix.

To demonstrate this outcome, we developed a new real-time fluorescence-based method for investigating the photocatalytic activity inside the hydrogels. One original feature of this approach is that the photocatalyst irradiation and the target degradation detection can be performed simultaneously in a standard fluorimeter, as shown in Figure 2.

\section{RESULTS AND DISCUSSION}

Hydrogel Preparation and Characterization. For the hydrogel preparation, we used the $\mathrm{pH}$ change method. This method relies upon the enhanced solubility of Fmoc-Tyr-Oxd$\mathrm{OH}$ at basic $\mathrm{pH}$, followed by a slow decrease of $\mathrm{pH}$ by the addition of glucono- $\delta$-lactone. ${ }^{41}$ We prepared three different samples of hydrogels: (i) hydrogel $\mathbf{1}(\mathbf{H})$ contains only the gelator Fmoc-L-Tyr-D-Oxd-OH in $0.5 \%(\mathrm{w} / \mathrm{w})$ concentration and glucono- $\delta$-lactone; (ii) hydrogel 2 (H-T) contains the gelator in $0.5 \%$ concentration, $\mathrm{TiO}_{2}-\mathrm{NPs}(0.2 \mathrm{mg} / \mathrm{mL})$, and glucono- $\delta$-lactone; (iii) 3 (H-TG) is prepared following the same procedure used for 2 (H-T), but replacing $\mathrm{TiO}_{2}-\mathrm{NPs}$ with $\mathrm{TiO}_{2}$-NPs/graphene. ${ }^{42}$

Before testing the hydrogels based on Fmoc-L-Tyr-D-Oxd$\mathrm{OH}$ for the photocatalytic applications, we investigated the effect of $\mathrm{TiO}_{2}-\mathrm{NPs}$ or $\mathrm{TiO}_{2}-\mathrm{NPs} /$ graphene on their mechanical and morphological characteristics. Rheological analyses have been performed to evaluate the viscoelastic properties of hydrogels $\mathbf{1}(\mathbf{H}), \mathbf{2}(\mathrm{H}-\mathrm{T})$, and $\mathbf{3}$ (H-TG) in terms of storage and loss moduli ( $G^{\prime}$ and $G^{\prime \prime}$, respectively) (Table land Figures S1 and S2).

Table 1. Storage Moduli $\left(G^{\prime}\right)$ and Loss Moduli $\left(G^{\prime \prime}\right)$ of Hydrogels 1 (H), 2 (H-T), and 3 (H-TG)

$\begin{array}{lcc}\text { hydrogel } & G^{\prime}(\mathrm{Pa}) & G^{\prime \prime}(\mathrm{Pa}) \\ \mathbf{1}(\mathbf{H}) & 1370 & 90 \\ \mathbf{2}(\mathbf{H}-\mathbf{T}) & 1055 & 63 \\ \text { 3 (H-TG) } & 556 & 46\end{array}$

Although the hydrogels are characterized by a "solidlike" behavior, that is, the storage modulus is approximately an order of magnitude higher than the loss component, these hydrogels do not show very high $G^{\prime}$ values because of the very low concentration of the gelator, required for obtaining good transparency. The results also pointed out that although the introduction of $\mathrm{TiO}_{2}-\mathrm{NPs}$ or $\mathrm{TiO}_{2}-\mathrm{NPs} /$ graphene partially affects the hydrogel properties, as in both cases, $G^{\prime}$ decreases. This effect is more evident for hydrogel 3 (H-TG). Nevertheless, frequency sweep analysis (Figure S2) pointed out that for all obtained hydrogels, both $G^{\prime}$ and $G^{\prime \prime}$ were almost independent of the frequency in the range from 0.1 to $100 \mathrm{rad} \cdot \mathrm{s}^{-1}$ (always with $G^{\prime}>G^{\prime \prime}$ ), confirming the "solidlike" rheological behavior of the hydrogels and hence the stability of the hydrogel structure required for the photocatalytic application.

Step-strain experiments were performed to check the thixotropic behavior of $\mathbf{1}(\mathbf{H}), \mathbf{2}(\mathbf{H}-\mathrm{T})$, and $\mathbf{3}$ (H-TG) on the molecular level. Strain values within and above the LVE (linear viscoelastic range) region were consecutively applied to the hydrogels, which lose their "solidlike" behavior $\left(G^{\prime}<G^{\prime \prime}\right)$ when the strain is applied above their LVE region and quickly go back to a "solidlike" state $\left(G^{\prime}>G^{\prime \prime}\right)$ if the strain is applied in the LVE region of the hydrogels (Figure 3). The three hydrogels show a thixotropic behavior, even though the $G^{\prime}$ and $G^{\prime \prime}$ values of the graphene-doped hydrogel 3 (H-TG) are lower than the $\mathbf{1}(\mathbf{H})$ and $2(\mathbf{H}-\mathbf{T}) G^{\prime}$ and $G^{\prime \prime}$ values.

More information on the nature of hydrogels $\mathbf{1}(\mathbf{H}), \mathbf{2}(\mathbf{H}-$ T), and 3 (H-TG) was obtained by scanning electron microscopy (SEM) analysis of the corresponding aerogels obtained by freeze-drying these samples (Figure 4). These aerogels show a different morphology, but they are all characterized by complex patterns with a rough orientation. Moreover, in aerogel 3 (H-TG) (Figure 4C and magnification), we can notice the presence of some small aggregates responsible for the formation of a less homogeneous hydrogel, in agreement with the results obtained from the rheological experiments.

Photodegradation Experiments. As mentioned, here, we propose an experimental approach based on fluorescence detection for investigating the photodegradation of a target molecule in the gel. As a main difference with respect to other 

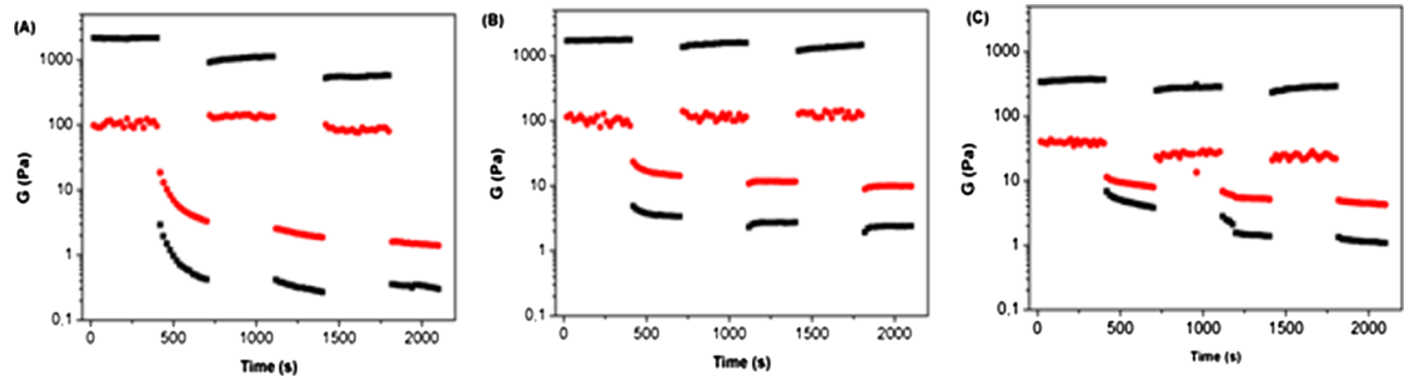

Figure 3. Values of storage moduli $G^{\prime}$ (black) and loss moduli $G^{\prime \prime}$ (red) recorded during a step-strain experiment performed on (A) hydrogel 1 (H), (B) hydrogel 2 (H-T), and (C) hydrogel 3 (H-TG).
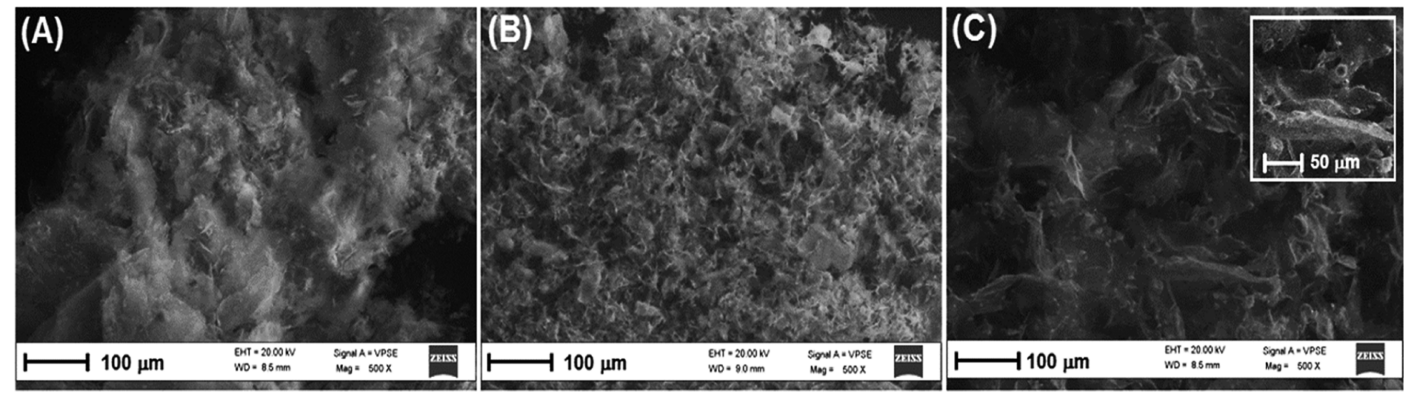

Figure 4. (A) SEM image of a sample of aerogel obtained by freeze-drying a sample of hydrogels $\mathbf{1}$ (H). (B) SEM image of a sample of aerogel obtained by freeze-drying a sample of hydrogels 2 (H-T). (C) SEM image of a sample of aerogel obtained by freeze-drying a sample of hydrogels and 3 (H-TG) prepared with Fmoc-L-Tyr-D-Oxd-OH 0.5\% concentration. In the inset, a magnification view of aerogel film fragments.

methods based on fluorescence, we propose to use the same excitation beam and the same excitation wavelength for both photoactivating the photocatalyst $\mathrm{TiO}_{2}$ and exciting the target fluorophore $\mathbf{R h B}$ (see Figure 2). As a main advantage, this approach allows the continuous real-time detection of the target dye concentration during the process using a conventional fluorimeter equipped with a $150 \mathrm{~W}$ xenon lamp as an excitation source.

To select a suitable irradiation wavelength, we analyzed the absorption spectra of hydrogel $\mathbf{1}(\mathbf{H})$ of the $\mathrm{TiO}_{2}$ - NPs $(0.2$ $\mathrm{mg} / \mathrm{mL})$ and $\mathrm{RhB}(1.0 \mu \mathrm{M})$ in a cuvette with a $0.5 \mathrm{~cm}$ optical path (spectra are shown in Figure 5). These spectra demonstrate that it is possible to excite simultaneously the $\mathrm{TiO}_{2}-\mathrm{NPs}$ and $\mathrm{RhB}$ upon irradiation in the UV spectral region

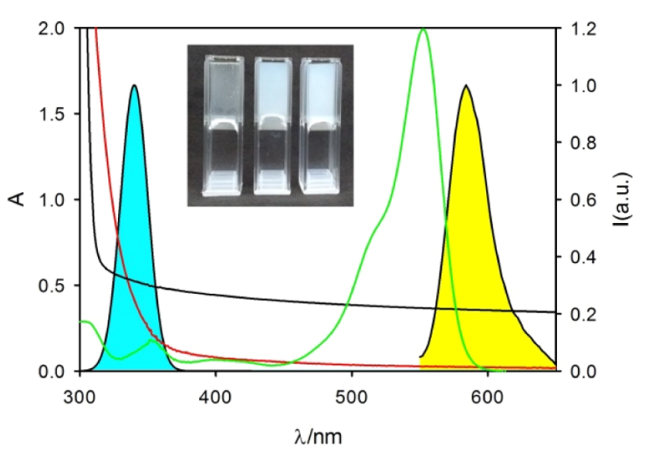

Figure 5. Absorption spectrum of hydrogel $1(\mathrm{H})$ (black line) of the $\mathrm{TiO}_{2} \mathrm{NPs}(0.2 \mathrm{mg} / \mathrm{mL}$, red line $)$ and $\mathrm{RhB}(\times 20,1 \mu \mathrm{M}$, green line $)$. Fluorescence spectrum of RhB (filled yellow) and spectrum of the irradiation source (filled cyan). Inset: photographs of samples of hydrogels 1 (H), 2 (H-T), and 3 (H-TG) (from left to right, respectively) prepared with gelator Fmoc-L-Tyr-D-Oxd-OH in $0.5 \%$ concentration. when they are incorporated in the hydrogel (as sketched in Figure 2).

In fact, as foreseen, the hydrogel presents an edge of absorption around $310 \mathrm{~nm}$ because of the characteristic absorption spectra of the Fmoc chromophore ${ }^{26}$ that is present in the gelator structure. ${ }^{10,43,44}$ The hydrogel transmittance is $T$ $>60 \%$ for wavelength $\lambda>320 \mathrm{~nm}$, and hence the photocatalyst $\mathrm{TiO}_{2}$-NPs incorporated in the hydrogel in $\mathbf{1}(\mathbf{H})$ can be efficiently excited at $340 \pm 10 \mathrm{~nm}$ using the fluorimeter excitation beam as an irradiation source (the spectral profile is shown in Figure 5).

Additionally, under these excitation conditions, RhB absorbs a minor fraction of the excitation light, and its fluorescence can be exploited as a diagnostic signal to follow the photodegradation of this dye during the photocatalytic experiments.

To investigate the $\mathrm{TiO}_{2}$ photocatalytic activity in the hydrogel, we prepared three samples like $\mathbf{1}(\mathbf{H}), 2$ (H-T), and 3 (H-TG), all containing a small concentration $(1.0 \mu \mathrm{M})$ of RhB. For clarity, these samples will be referred as 4 (HR), 5 (HR-T), and 6 (HR-TG). Hydrogel rheological properties were not affected by the presence of small concentrations of RhB $(1.0 \mu \mathrm{M})$ in the hydrogels.

Results, reported in Figure 6, show that there is a strong decrease in $\mathbf{R h B}$ emission during the first hour of irradiation in the case of 5 (HR-T) and 6 (HR-TG). By contrast, in the reference hydrogel 4 (HR), the fluorescence signal decreases only by about $20 \%$ during the first $10 \mathrm{~min}(600 \mathrm{~s})$, and then it reaches a plateau. ${ }^{45,46}$ Hence, as the fluorescence intensity is proportional to the fluorophore concentration, under these experimental conditions, ${ }^{47,48}$ we can gather that after $60 \mathrm{~min}$ of irradiation, the $\mathbf{R h B}$ concentration in the reference sample 4 (HR) is still $80 \%$ of the initial one, whereas the dye is completely degraded in the $\mathrm{TiO}_{2}$-containing sample 5 (HR-T) and a residual $25 \%$ survived in 6 (HR-TG). These results clearly demonstrate that (i) the $\mathrm{TiO}_{2}$-NPs photodecompose 


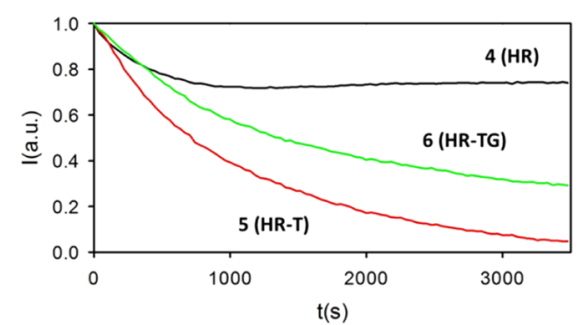

Figure 6. Fluorescence intensity at $585 \mathrm{~nm}$ of $\mathrm{RhB}$ as a function of irradiation time $\left(\lambda_{\text {exc }}=340 \mathrm{~nm}\right)$ in sample $4($ HR), 5 (HR-T), and 6 (HR-TG).

efficiently in $\mathbf{R h B}$, but (ii) the introduction of graphene in hydrogel 6 (HR-TG) leads to a photoactivity decrease of $\mathrm{TiO}_{2}$-NPs, in contrast with what was observed in other matrixes. ${ }^{49}$

Going into more detail, a simple parameter suitable to quantify the rate of the photodegradation process is the halflife $t_{1 / 2}$, which is defined as the irradiation time at which the concentration value of the reactant $(\mathrm{RhB})$ becomes half of the initial one. The half-life $t_{1 / 2}$ of $\mathbf{R h B}$ corresponds to 12 and 23 min of irradiation for 5 (HR-T) and 6 (HR-TG), respectively.

To go into more details of the photodegradation kinetics, we analyzed the kinetics traces of Figure 6 according to a (pseudo) first-order model (see the Supporting Information). From the data analysis, we obtain values of the rate constant $k$ $=8.0 \pm 0.1 \times 10^{-4} \mathrm{~s}^{-1}$ and $k=4.0 \pm 0.1 \times 10^{-4} \mathrm{~s}^{-1}$ for 5 (HRT) and 6 (HR-TG), respectively. This result further confirms that the presence of graphene in the hydrogel apparently causes a decrease in the $\mathrm{TiO}_{2}$-NP photocatalytic activity. Indeed, SEM and rheological analyses clearly indicate that graphene affects the matrix morphology and causes a homogeneity decrease of the $\mathrm{TiO}_{2}-\mathrm{NP}$ dispersion (see the Supporting Information): this alteration is the actual reason for the decreased photocatalytic activity of 6 (HR-TG) when compared to 5 (HR-T).

As far as the mechanism of photodegradation is concerned, the observed (pseudo) first-order kinetics is consistent with the Langmuir-Hinshelwood model, which is the most representative in describing the kinetic degradation of xanthene dyes as RhB in heterogeneous photocatalysis. This model takes into consideration the contribution of both dye adsorption on the photocatalyst surface and dye diffusion. ${ }^{50}$

In view of future applications, it is very important to understand whether, in the hydrogel, the target molecules RhB can freely diffuse or they are bound either to the gelator fibers or to the $\mathrm{TiO}_{2}-\mathrm{NPs}$. Free diffusion of the pollutant moiety is in fact essential for the development of a material suitable for continuous recyclable materials for water decontamination.

We hence investigated the interactions between the target dye molecules and the microenvironment by steady-state and time-resolved fluorescence spectroscopies, using fluorescence anisotropy to measure the local mobility of $\mathrm{RhB}$ in 4 (HR), 5 (HR-T), and 6 (HR-TG).

Quenching of RhB fluorescence has been reported to occur upon absorption on $\mathrm{TiO}_{2}$ or onto the honeycomb carbon structure of graphene. ${ }^{51,52}$ Hence, we recorded and compared the fluorescence emission and excitation spectra of $\mathrm{RhB}\left(\lambda_{\mathrm{exc}}=\right.$ $530 \mathrm{~nm}$ ) of 4 (HR), 5 (HR-T), and 6 (HR-TG). On the basis of the fluorescence spectra, shown in Figure 7, we concluded that no quenching of the fluorescence of RhB could be observed in 5 (HR-T) and 6 (HR-TG).

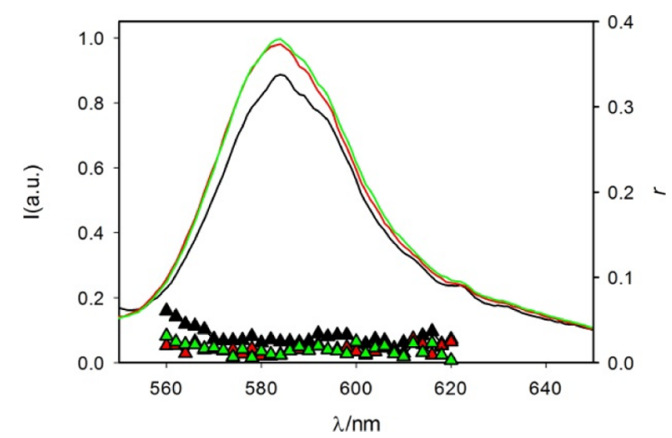

Figure 7. Fluorescence spectra $\left(\lambda_{\text {exc }}=530 \mathrm{~nm}\right)$ of RhB in sample 4 (HR) (black line), 5 (HR-T) (red line), and 6 (HR-TG) (green line) and fluorescence anisotropy of the same samples: black, red, and green triangles, respectively.

This conclusion was further supported by time-resolved fluorescence measurements. Excited-state lifetimes were measured by time-correlated single photon counting. Fluorescence decays recorded for RhB in 4 (HR), 5 (HR-T), and 6 (HR-TG) were fitted with a single exponential model to give excited-state lifetimes of $3.4,3.7$, and 3.6 ns, respectively. These values match with the lifetime of the fluorophore in water solution, confirming that no quenching of the fluorescence of the target dye occurs in 4 (HR), 5 (HR-T), and $\mathbf{6}$ (HR-TG). ${ }^{48,53,54}$

As mentioned, fluorescence anisotropy ( $r$, dimensionless) measurements allow to determine the rotational mobility of a fluorophore in a given environment. Values of $r$ close to 0.4 were reported for rhodamine molecules in the case of strongly hindered rotation, ${ }^{55,56}$ whereas the same molecules have an $r$ very close to 0 when free to diffuse in poorly viscous solvent such as water.

Fluorescence anisotropy spectra of $\mathrm{RhB}$ in 4 (HR), 5 (HRT), and 6 (HR-TG) are shown in Figure 7 (triangles); in all three cases, the anisotropy around the maximum fluorescence is very low $(0.02-0.03)$ and similar to the value measured in pure water. This result confirms that the dye molecules can diffuse fast in the hydrogel (hence, the diffusion time is much shorter than the excited-state lifetime) and are dissolved in the hydrogel water channels rather than adsorbed on the gelator fibers.

\section{CONCLUSIONS}

In this paper, we reported the incorporation of $\mathrm{TiO}_{2}-\mathrm{NPs}$ (also in combination with graphene platelets) into highly biocompatible hydrogels, whose properties have been analyzed by rheological and SEM analyses.

These hydrogels show a high water content and good transparency to solar light, and they degrade a pollutant model molecule with good efficiency upon semiconductor NP irradiation.

Interestingly, our experiments demonstrate that light penetrates inside the hydrogel and that photodegradation occurs in the bulk of the material. This important result was achieved by optimizing the composition and the methodology of production of the hydrogel to avoid sedimentation and segregation of the $\mathrm{TiO}_{2}$-NPs (as demonstrated by the SEM analysis).

We also proved that while photodegradation of the target molecule (pollutant model) efficiently occurs upon irradiation, the photogenerated reactive species does not alter the matrix 
properties: rheology of the hydrogel, in fact, is not modified either by the incorporation of the photocatalysts or upon their irradiation.

Finally, we investigated the actual mobility of the pollutant molecules in the hydrogel on the microscopic level by steadystate and time-resolved fluorescence anisotropy spectroscopies. Our results demonstrated that these molecules are not absorbed either on the hydrogel fibers or on the $\mathrm{TiO}_{2}-\mathrm{NPs}$, suggesting that pollutant molecules are rather solubilized in the hydrogel water channel. This result is very important in view of the development of photocatalytic water-permeable materials for continuous decontamination of water in fluxing systems.

\section{EXPERIMENTAL SECTION}

Materials. All chemicals and solvents were purchased from Sigma-Aldrich, VWR or Iris Biotech and were used as received. Acetonitrile was distilled under an inert atmosphere before use. Milli-Q water (Millipore, resistivity $=18.2 \mathrm{~m} \Omega \cdot \mathrm{cm}$ ) was used throughout.

Synthesis of Fmoc-L-Tyr-D-Oxd-OH. The compound was synthesized from D-Thr and Fmoc-L-Tyr $(t-\mathrm{Bu})-\mathrm{OH}$ following a multistep procedure in solution, reported in ref 24 .

Hydrogel Preparation. A portion of Fmoc-L-Tyr-D-Oxd$\mathrm{OH}(5 \mathrm{mg})$ was placed in a test tube (diameter: $8 \mathrm{~mm}$ ); then Milli-Q water $(0.97 \mathrm{~mL})$ and a $1 \mathrm{M}$ aqueous $\mathrm{NaOH}(1.3$ equiv) were added; and the mixture was stirred until sample dissolution. Finally, we added 1.4 equiv of glucono- $\delta$-lactone, and the mixture was left still for $16 \mathrm{~h}$ at room temperature. For more details, see Scheme S1.

Aerogel Preparation. Some samples of hydrogels 1-3 were freeze-dried using a BENCHTOP Freeze Dry System LABCONCO 7740030 with the following procedure: The hydrogel was prepared into an Eppendorf test tube at room temperature. After $16 \mathrm{~h}$, the samples were deepened in liquid nitrogen for $10 \mathrm{~min}$ and then freeze-dried for $24 \mathrm{~h}$ in vacuo $(0.2 \mathrm{mBar})$ at $-50{ }^{\circ} \mathrm{C}$.

Hydrogel Characterization. Morphological Analysis. Scanning electron micrographs of the samples were recorded using a Zeiss EP EVO 50 field emission gun scanning electron microscope. Conditions: EHT $=20 \mathrm{keV}$ - variable pressure: $100 \mathrm{~Pa}$-images in secondary electrons.

Rheology. Rheology experiments were carried out on an Anton Paar rheometer MCR 102 using parallel plate configuration (25 mm diameter). Experiments were performed at a constant temperature of $23^{\circ} \mathrm{C}$ controlled by the integrated Peltier system and a Julabo AWC100 cooling system.

Ultraviolet-Visible (UV-Vis) Absorption Spectra. UV-vis absorption spectra (range 200-800 nm) were collected by using an optical path of $0.5 \mathrm{~cm}$ cuvette at 25 with a Cary 300 $\mathrm{UV}$-vis double beam spectrophotometer, having an empty cuvette as a reference.

Steady-State and Time-Resolved Fluorescence Spectroscopies and Fluorescence Anisotropy. Fluorescence spectra were collected with an Edinburgh FLS920 fluorimeter equipped with a photomultiplier Hamamatsu R928P, and the samples were analyzed in disposable cuvettes with an optical path length of $0.5 \mathrm{~cm}$. Emission spectra: $\lambda_{\mathrm{exc}}=530 \mathrm{~nm} ; \lambda_{\mathrm{em}}$ : $540-700 \mathrm{~nm}$. Excitation spectra: $\lambda_{\mathrm{exc}}=330-600 \mathrm{~nm}, \lambda_{\mathrm{em}}=$ $620 \mathrm{~nm}$. Fluorescence anisotropy: $\lambda_{\mathrm{exc}}=530 \mathrm{~nm} ; \lambda_{\mathrm{em}}: 550-$ $650 \mathrm{~nm}$.

Photodegradation Experiments. The kinetic analysis was carried on by continuously monitoring the degradation of RhB acquiring the fluorescence spectra with a Horiba FluoroMax-4 spectrofluorimeter upon excitation/irradiation at $340 \mathrm{~nm}$ (10 $\mathrm{nm}$ slits). Experimental parameters are reported in detail in Scheme S2.

\section{ASSOCIATED CONTENT}

\section{Supporting Information}

The Supporting Information is available free of charge on the ACS Publications website at DOI: 10.1021/acsomega.8b01037.

Preparation and characterization of hydrogels $\mathbf{1}(\mathrm{H}), \mathbf{2}$ (H-T), 1 (H), 2 (H-T), 4 (HR), 5 (HR-T), and 6 (HRTG), strain dependence and frequency dependence for hydrogels 1-3, emission spectra and excitation spectra of 1 (H), 2 (H-T), 4 (HR), 5 (HR-T), and 6 (HR-TG), comparison of the RhB emission lifetime decays in the ns timescale for 4 (HR), 5 (HR-T), and 6 (HR-TG), fluorescence anisotropy measurements for the samples 4 (HR), 5 (HR-T), and 6 (HR-TG) and hypothesis of the mechanism of the RhB degradation pathway (PDF)

\section{AUTHOR INFORMATION}

\section{Corresponding Authors}

*E-mail: marco.montalti2@unibo.it (M.M.)

*E-mail: claudia.tomasini@unibo.it (C.T.).

ORCID

Matteo Calvaresi: 0000-0002-9583-2146

Claudia Tomasini: 0000-0002-6310-2704

Notes

The authors declare no competing financial interest.

\section{ACKNOWLEDGMENTS}

We gratefully acknowledge Ministero dell'Università e della Ricerca (PRIN 2015 project 20157WW5EH) and Alma Mater Studiorum Università di Bologna for the financial support. This project has received funding from the European Union's Horizon 2020 research and innovation programme under grant agreement no. 696656 (Graphene Flagship).

\section{REFERENCES}

(1) Okesola, B. O.; Smith, D. K. Applying low-molecular weight supramolecular gelators in an environmental setting - self-assembled gels as smart materials for pollutant removal. Chem. Soc. Rev. 2016, 45, $4226-4251$.

(2) Nosaka, Y.; Nosaka, A. Y. Generation and Detection of Reactive Oxygen Species in Photocatalysis. Chem. Rev. 2017, 117, 1130211336.

(3) Tong, H.; Ouyang, S.; Bi, Y.; Umezawa, N.; Oshikiri, M.; Ye, J. Nano-photocatalytic Materials: Possibilities and Challenges. Adv. Mater. 2012, 24, 229-251.

(4) Fleisch, M.; Bahnemann, D. Photokatalytisch aktiver Beton: Wie innovative Baustoffe einen Beitrag zum Abbau gefährlicher Luftschadstoffe leisten können. Beton-Stahlbetonbau 2017, 112, 47-53.

(5) Lai, Y.; Huang, J.; Cui, Z.; Ge, M.; Zhang, K.-Q.; Chen, Z.; Chi, L. Recent Advances in TiO2-Based Nanostructured Surfaces with Controllable Wettability and Adhesion. Small 2016, 12, 2203-2224.

(6) Nishimoto, S.; Bhushan, B. Bioinspired Self-Cleaning Surfaces with Superhydrophobicity, Superoleophobicity, and Superhydrophilicity. RSC Adv. 2013, 3, 671-690.

(7) Mamaghani, A. H.; Haghighat, F.; Lee, C.-S. Photocatalytic Oxidation Technology for Indoor Environment Air Purification: The State-of-the-Art. Appl. Catal., B 2017, 203, 247-269.

(8) Yu, C.; Zhou, W.; Liu, H.; Liu, Y.; Dionysiou, D. D. Design and Fabrication of Microsphere Photocatalysts for Environmental 
Purification and Energy Conversion. Chem. Eng. J. 2016, 287, 117129.

(9) Xia, T.; Kovochich, M.; Brant, J.; Hotze, M.; Sempf, J.; Oberley, T.; Sioutas, C.; Yeh, J. I.; Wiesner, M. R.; Nel, A. E. Comparison of the Abilities of Ambient and Manufactured Nanoparticles to Induce Cellular Toxicity According to an Oxidative Stress Paradigm. Nano Lett. 2006, 6, 1794-1807.

(10) Hoffmann, M. R.; Martin, S. T.; Choi, W.; Bahnemann, D. W. Environmental Applications of Semiconductor Photocatalysis. Chem. Rev. 1995, 95, 69-96.

(11) Vale, G.; Mehennaoui, K.; Cambier, S.; Libralato, G.; Jomini, S.; Domingos, R. F. Manufactured Nanoparticles in the Aquatic Environment-Biochemical Responses on Freshwater Organisms: A Critical Overview. Aquat. Toxicol. 2016, 170, 162-174.

(12) Djurišić, A. B.; Leung, Y. H.; Ng, A. M. C.; Xu, X. Y.; Lee, P. K. H.; Degger, N.; Wu, R. S. S. Toxicity of Metal Oxide Nanoparticles: Mechanisms, Characterization, and Avoiding Experimental Artefacts. Small 2014, 11, 26-44.

(13) Smith, D. K. Building bridges. Nat. Chem. 2010, 2, 162-163.

(14) Amabilino, D. B.; Smith, D. K.; Steed, J. W. Supramolecular Materials. Chem. Soc. Rev. 2017, 46, 2404-2420.

(15) Montalti, M.; Dolci, L. S.; Prodi, L.; Zaccheroni, N.; Stuart, M. C. A.; van Bommel, K. J. C.; Friggeri, A. Energy Transfer from a Fluorescent Hydrogel to a Hosted Fluorophore. Langmuir 2006, 22, 2299-2303.

(16) Hahn, M. E.; Gianneschi, N. C. Enzyme-Directed Assembly and Manipulation of Organic Nanomaterials. Chem. Commun. 2011, 47, 11814-11821.

(17) Zanna, N.; Focaroli, S.; Merlettini, A.; Gentilucci, L.; Teti, G.; Falconi, M.; Tomasini, C. Thixotropic Peptide-Based Physical Hydrogels Applied to Three-Dimensional Cell Culture. ACS Omega 2017, 2, 2374-2381.

(18) Tomasini, C.; Zanna, N. Oxazolidinone-Containing Pseudopeptides: Supramolecular Materials, Fibers, Crystals, and Gels. Biopolymers 2017, 108, No. e22898.

(19) Zanna, N.; Merlettini, A.; Tomasini, C. Self-Healing Hydrogels Triggered by Amino Acids. Org. Chem. Front. 2016, 3, 1699-1704.

(20) Castellucci, N.; Falini, G.; Angelici, G.; Tomasini, C. Formation of Gels in the Presence of Metal Ions. Amino Acids 2011, 41, 609620.

(21) Milli, L.; Castellucci, N.; Tomasini, C. Turning Around theLPhe-D-Oxd Moiety for a Versatile Low-Molecular-Weight Gelator. Eur. J. Org. Chem. 2014, 5954-5961.

(22) Dong, C.; Lu, J.; Qiu, B.; Shen, B.; Xing, M.; Zhang, J. Developing Stretchable and Graphene-Oxide-Based Hydrogel for the Removal of Organic Pollutants and Metal Ions. Appl. Catal., B 2018, $222,146-156$.

(23) Zhu, H.; Li, Z.; Yang, J. A Novel Composite Hydrogel for Adsorption and Photocatalytic Degradation of Bisphenol A by Visible Light Irradiation. Chem. Eng. J. 2018, 334, 1679-1690.

(24) Zanna, N.; Merlettini, A.; Tatulli, G.; Milli, L.; Focarete, M. L.; Tomasini, C. Hydrogelation Induced by Fmoc-Protected Peptidomimetics. Langmuir 2015, 31, 12240-12250.

(25) Liyanage, W.; Vats, K.; Rajbhandary, A.; Benoit, D. S. W.; Nilsson, B. L. Multicomponent Dipeptide Hydrogels as Extracellular Matrix-Mimetic Scaffolds for Cell Culture Applications. Chem. Commun. 2015, 51, 11260-11263.

(26) Tao, K.; Levin, A.; Adler-Abramovich, L.; Gazit, E. FmocModified Amino Acids and Short Peptides: Simple Bio-Inspired Building Blocks for the Fabrication of Functional Materials. Chem. Soc. Rev. 2016, 45, 3935-3953.

(27) Orbach, R.; Mironi-Harpaz, I.; Adler-Abramovich, L.; Mossou, E.; Mitchell, E. P.; Forsyth, V. T.; Gazit, E.; Seliktar, D. The Rheological and Structural Properties of Fmoc-Peptide-Based Hydrogels: The Effect of Aromatic Molecular Architecture on Self-Assembly and Physical Characteristics. Langmuir 2012, 28, 2015-2022.

(28) Raeburn, J.; Mendoza-Cuenca, C.; Cattoz, B. N.; Little, M. A.; Terry, A. E.; Zamith Cardoso, A.; Griffiths, P. C.; Adams, D. J. The effect of solvent choice on the gelation and final hydrogel properties of Fmoc-diphenylalanine. Soft Matter 2015, 11, 927-935.

(29) Linsebigler, A. L.; Lu, G.; Yates, J. T., Jr Photocatalysis on TiO2 Surfaces: Principles, Mechanisms, and Selected Results. Chem. Rev. 1995, 95, 735-758.

(30) Ciesielski, A.; Samorì, P. Grapheneviasonication assisted liquidphase exfoliation. Chem. Soc. Rev. 2014, 43, 381-398.

(31) Dai, K.; Peng, T.; Ke, D.; Wei, B. Photocatalytic hydrogen generation using a nanocomposite of multi-walled carbon nanotubes and $\mathrm{TiO} 2$ nanoparticles under visible light irradiation. Nanotechnology 2009, 20, 125603.

(32) Sun, M.; Ma, X.; Chen, X.; Sun, Y.; Cui, X.; Lin, Y. A nanocomposite of carbon quantum dots and $\mathrm{TiO} 2$ nanotube arrays: enhancing photoelectrochemical and photocatalytic properties. RSC Adv. 2014, 4, 1120-1127.

(33) Reddy, K. R.; Hassan, M.; Gomes, V. G. Hybrid Nanostructures Based on Titanium Dioxide for Enhanced Photocatalysis. Appl. Catal., A 2015, 489, 1-16.

(34) Han, L.; Wang, P.; Dong, S. Progress in Graphene-Based Photoactive Nanocomposites as a Promising Class of Photocatalyst. Nanoscale 2012, 4, 5814-5825.

(35) Mukherjee, A.; Chakrabarty, S.; Kumari, N.; Su, W.-N.; Basu, S. Visible-Light-Mediated Electrocatalytic Activity in Reduced Graphene Oxide-Supported Bismuth Ferrite. ACS Omega 2018, 3, 5946-5957.

(36) Serpone, N.; Lawless, D.; Khairutdinov, R. Size Effects on the Photophysical Properties of Colloidal Anatase TiO2 Particles: Size Quantization versus Direct Transitions in This Indirect Semiconductor? J. Phys. Chem. 1995, 99, 16646-16654.

(37) Kathiravan, A.; Renganathan, R. Effect of Anchoring Group on the Photosensitization of Colloidal $\mathrm{TiO} 2$ Nanoparticles with Porphyrins. J. Colloid Interface Sci. 2009, 331, 401-407.

(38) Folli, A.; Jakobsen, U. H.; Guerrini, G. L.; Macphee, D. E. Rhodamine B Discolouration on $\mathrm{TiO} 2$ in the Cement Environment: A Look at Fundamental Aspects of the Self-Cleaning Effect in Concretes. J. Adv. Oxid. Technol. 2009, 12, 126-133.

(39) Folli, A.; Pade, C.; Hansen, T. B.; De Marco, T.; Macphee, D. E. TiO2 Photocatalysis in Cementitious Systems: Insights into SelfCleaning and Depollution Chemistry. Cem. Concr. Res. 2012, 42, $539-548$

(40) Zailan, S. N.; Mahmed, N.; Abdullah, M. M. A.; Victor Sandu, A.; Shahedan, N. F. Review on Characterization and Mechanical Performance of Self-Cleaning Concrete. In Engineering Technology International Conference 2016; Abdullah, M. A. B., AbdRahim, S. Z., Suandi, M. E. M., Saad, M. N. M., Ghazali, M. F., Eds.; MATEC Web of Conferences, 2017; Vol. 97.

(41) Adams, D. J.; Butler, M. F.; Frith, W. J.; Kirkland, M.; Mullen, L.; Sanderson, P. A new method for maintaining homogeneity during liquid-hydrogel transitions using low molecular weight hydrogelators. Soft Matter 2009, 5, 1856-1862.

(42) Milli, L.; Zanna, N.; Merlettini, A.; Di Giosia, M.; Calvaresi, M.; Focarete, M. L.; Tomasini, C. Pseudopeptide-Based Hydrogels Trapping Methylene Blue and Eosin Y. Chem.-Eur. J. 2016, 22, 12106-12112.

(43) Haiss, W.; Thanh, N. T. K.; Aveyard, J.; Fernig, D. G. Determination of Size and Concentration of Gold Nanoparticles from UV-Vis Spectra. Anal. Chem. 2007, 79, 4215-4221.

(44) Trapalis, A.; Todorova, N.; Giannakopoulou, T.; Boukos, N.; Speliotis, T.; Dimotikali, D.; Yu, J. TiO 2 /graphene composite photocatalysts for NOx removal: A comparison of surfactantstabilized graphene and reduced graphene oxide. Appl. Catal., B 2016, 180, 637-647.

(45) Zondervan, R.; Kulzer, F.; Kol'chenk, M. A.; Orrit, M. Photobleaching of Rhodamine 6G in Poly(Vinyl Alcohol) at the Ensemble and Single-Molecule Levels. J. Phys. Chem. A 2004, 108, $1657-1665$.

(46) Wilhelm, P.; Stephan, D. Photodegradation of Rhodamine B in Aqueous Solution via SiO2@TiO2 Nano-Spheres. J. Photochem. Photobiol., A 2007, 185, 19-25. 
(47) Montalti, M.; Credi, A.; Prodi, L.; Gandolfi, M. T. Handbook of Photochemistry, 3rd ed.; CRC Press, 2006.

(48) Genovese, D.; Bonacchi, S.; Juris, R.; Montalti, M.; Prodi, L.; Rampazzo, E.; Zaccheroni, N. Prevention of Self-Quenching in Fluorescent Silica Nanoparticles by Efficient Energy Transfer. Angew. Chem., Int. Ed. 2013, 52, 5965-5968.

(49) Dahl, M.; Liu, Y.; Yin, Y. Composite Titanium Dioxide Nanomaterials. Chem. Rev. 2014, 114, 9853-9889.

(50) Rajeshwar, K.; Osugi, M. E.; Chanmanee, W.; Chenthamarakshan, C. R.; Zanoni, M. V. B.; Kajitvichyanukul, P.; Krishnan-Ayer, R. Heterogeneous Photocatalytic Treatment of Organic Dyes in Air and Aqueous Media. J. Photochem. Photobiol., C 2008, 9, 171-192.

(51) Hsiao, Y.-C.; Wu, T.-F.; Wang, Y.-S.; Hu, C.-C.; Huang, C. Evaluating the sensitizing effect on the photocatalytic decoloration of dyes using anatase-TiO2. Appl. Catal., B 2014, 148-149, 250-257.

(52) Guidetti, G.; Cantelli, A.; Mazzaro, R.; Ortolani, L.; Morandi, V.; Montalti, M. Tracking Graphene by Fluorescence Imaging: A Tool for Detecting Multiple Populations of Graphene in Solution. Nanoscale 2016, 8, 8505-8511.

(53) Montalti, M.; Battistelli, G.; Cantelli, A.; Genovese, D. PhotoTunable Multicolour Fluorescence Imaging Based on Self-Assembled Fluorogenic Nanoparticles. Chem. Commun. 2014, 50, 5326.

(54) Bonacchi, S.; Cantelli, A.; Battistelli, G.; Guidetti, G.; Calvaresi, M.; Manzi, J.; Gabrielli, L.; Ramadori, F.; Gambarin, A.; Mancin, F.; Montalti, M. Photoswitchable NIR-Emitting Gold Nanoparticles. Angew. Chem., Int. Ed. 2016, 55, 11064-11068.

(55) Rampazzo, E.; Bonacchi, S.; Juris, R.; Montalti, M.; Genovese, D.; Zaccheroni, N.; Prodi, L.; Rambaldi, D. C.; Zattoni, A.; Reschiglian, P. Energy Transfer from Silica Core-Surfactant Shell Nanoparticles to Hosted Molecular Fluorophores. J. Phys. Chem. B 2010, 114, 14605-14613.

(56) Bonacchi, S.; Rampazzo, E.; Montalti, M.; Prodi, L.; Zaccheroni, N.; Mancin, F.; Teolato, P. Amplified Fluorescence Response of Chemosensors Grafted onto Silica Nanoparticles. Langmuir 2008, 24, 8387-8392. 